Mapping the lonization State of Laser-Irradiated Ar Gas Jets With Multi-Wavelength Monochromatic X-Ray Imaging

N. L. Kugland, T. Doppner, A. Kemp, D. Schaeffer, S. H. Glenzer, C. Niemann

April 22, 2010

Review of Scientific Instruments 
This document was prepared as an account of work sponsored by an agency of the United States government. Neither the United States government nor Lawrence Livermore National Security, LLC, nor any of their employees makes any warranty, expressed or implied, or assumes any legal liability or responsibility for the accuracy, completeness, or usefulness of any information, apparatus, product, or process disclosed, or represents that its use would not infringe privately owned rights. Reference herein to any specific commercial product, process, or service by trade name, trademark, manufacturer, or otherwise does not necessarily constitute or imply its endorsement, recommendation, or favoring by the United States government or Lawrence Livermore National Security, LLC. The views and opinions of authors expressed herein do not necessarily state or reflect those of the United States government or Lawrence Livermore National Security, LLC, and shall not be used for advertising or product endorsement purposes. 


\title{
Mapping the Ionization State of Laser-Irradiated Ar Gas Jets With Multi-Wavelength Monochromatic X-Ray Imaging
}

\author{
N. L. Kugland ${ }^{a, b}$, T. Döppner ${ }^{b}$, A. Kemp ${ }^{b}$, D. Schaeffer ${ }^{a}$, S. H. Glenzer ${ }^{b}$, and C. Niemann ${ }^{a, b}$ \\ a. Physics Department, University of California Los Angeles, Los Angeles, California, 90095, USA and \\ b. Lawrence Livermore National Laboratory, Livermore, California 94550, USA
}

\begin{abstract}
Two-dimensional monochromatic images of fast-electron stimulated $\mathrm{Ar} \mathrm{K} \alpha$ and $\mathrm{He}-\alpha$ x-ray self-emission have recorded a time-integrated map of the extent of $\mathrm{Ar}^{\approx 6+}$ and $\mathrm{Ar}^{16+}$ ions, respectively, within a high density $\left(10^{20} \mathrm{~cm}^{-3}\right.$ atomic density) Ar plasma. This plasma was produced by irradiating a $2 \mathrm{~mm}$ wide clustering Ar gas jet with an ultra-high intensity $\left(10^{19} \mathrm{~W} / \mathrm{cm}^{2}, 200 \mathrm{fs}\right)$ Ti:Sapphire laser operating at $800 \mathrm{~nm}$. Spherically bent quartz crystals in the 200 (for $\mathrm{K} \alpha$ ) and 201 (for $\mathrm{He}-\alpha$ ) planes were used as near-normal incidence reflective $\mathrm{x}$-ray optics. We see that a large ( $830 \mu \mathrm{m}$ long) region of plasma emits $\mathrm{K} \alpha$ primarily along the laser axis, while the He- $\alpha$ emission is confined to smaller hot spot $(230 \mu \mathrm{m}$ long $)$ region that likely corresponds to the focal volume of the f/8 laser beam. X-ray spectra from a Bragg spectrometer operating in the von Hamos geometry, which images in one dimension, indicate that the centroids of the $\mathrm{K} \alpha$ and $\mathrm{He}-\alpha$ emission regions are separated by approximately $330 \mu \mathrm{m}$ along the laser axis.
\end{abstract}

\section{INTRODUCTION}

Understanding the ionization dynamics of laser-produced plasmas is a research topic of fundamental interest in plasma physics. Ionization is caused by photon-particle and particleparticle interactions [1,2], and the ionization state of a plasma largely determines the nature of the dominant atomic physics processes, which in turn influence the plasma evolution [2-4]. For moderate and high-Z elements, the K-shell $\mathrm{x}$-ray lines correspond to relatively well defined ionic states $[5,6]$. Because these K-shell photon energies are sensitive to the ionization state of the emitting ion [5,7], imaging the self-emission of these $\mathrm{x}$-ray lines can provide a map of the corresponding ionization states within a plasma.

Especially where significant temperature gradients exist, such as inside inertial confinement fusion (ICF) plasmas, multi-wavelength monochromatic $x$-ray imaging (XRI) has been used as a plasma diagnostic. Specifically, imaging the $\mathrm{He}$ - and Ly- emission from an $\mathrm{Ar}$ or $\mathrm{Cl}$ dopant with timeresolved detectors can help document the evolution of capsule implosion and heating $[8,9]$. In general, by using an appropriate population level kinetics model, an ionization map can be used to infer plasma temperature $[10,11]$.

In this work we show that multi-wavelength monochromatic XRI can be used to map the time-integrated spatial distribution of ionization states in moderate temperature Ar plasmas that have been formed by ultra-short pulse laser irradiation of a super sonic Ar gas jet. We imaged both near-cold $\mathrm{K} \alpha$ x-rays [5, 12] at $h v=2965 \mathrm{eV}$, characteristic of $\mathrm{Ar} \approx 6+$ M-shell ions $\left(T_{e} \approx 20 \mathrm{eV}\right)$, as well as the He- $\alpha_{\text {res }} \mathrm{X}$-rays [13] at $h v=3140 \mathrm{eV}$ that are characteristic of $\mathrm{Ar}^{16+} \mathrm{K}$-shell ions $\left(T_{e} \gtrsim 100 \mathrm{eV}\right)$. In both cases, the $\mathrm{x}$-radiation is stimulated by laser-accelerated fast electron impact ionization. We observe a $230 \mu \mathrm{m}$ long region of $\mathrm{He}-\alpha_{\text {res }}$ emission, which corresponds to directly heated plasma where extensive ionization occurs due to strong laser-electron coupling. Centered at a distance of $330 \mu \mathrm{m}$ farther along the laser axis, we see a larger, $830 \mu \mathrm{m}$ long region of $\mathrm{K} \alpha$ emission. Observing the spatial extent of these two ionization states provides fundamental information about the nature of the laser-target interaction.

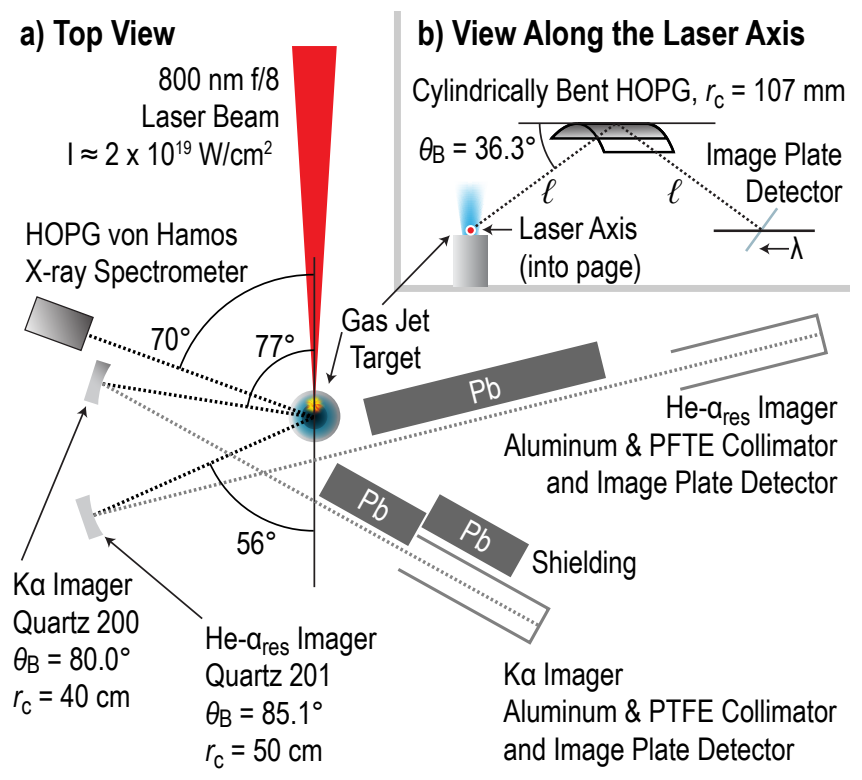

FIG. 1: (Color online) a) Top view of the experimental setup, showing placement of the gas jet target, $\mathrm{x}$-ray spectrometer, and the two $\mathrm{x}$-ray imagers. The gas jet fired upwards (i.e. out of the page), and the diagnostics were mostly arranged in the horizontal laser-target plane. Three lead shielding bricks were placed to block the lines of sight from the plasma to the XRI detectors.

\section{EXPERIMENTAL SETUP}

We used the Callisto laser at Lawrence Livermore National Laboratory, a Ti:Sapphire laser that provides up to $10 \mathrm{~J}$ of $\lambda=$ $800 \mathrm{~nm}$ light in $200 \mathrm{fs}$. With a typical focal spot radius of $r_{0}$ $=9 \mu \mathrm{m}$, the on-target intensity was approximately $2 \times 10^{19}$ $\mathrm{W} / \mathrm{cm}^{2}$. The target was a high-density super sonic clustering Ar gas jet, the details of which are described in detail in Ref. [14]. Near the nozzle exit, the characteristic atomic density of the gas jet was $1 \times 10^{20} \mathrm{~cm}^{-3}$. The target and the $\mathrm{x}$-ray diagnostics were arranged inside the laser target chamber as shown in Fig. 1.

An x-ray spectrometer monitored the source x-ray spectrum using a highly oriented pyrolytic graphite (HOPG) crystal $[15,16]$ that was cylindrically bent with a radius of curva- 
a) Ka X-ray Image (Corresponding with $\mathrm{Ar} \approx 6+$ M-Shell lons) $\vec{x}$
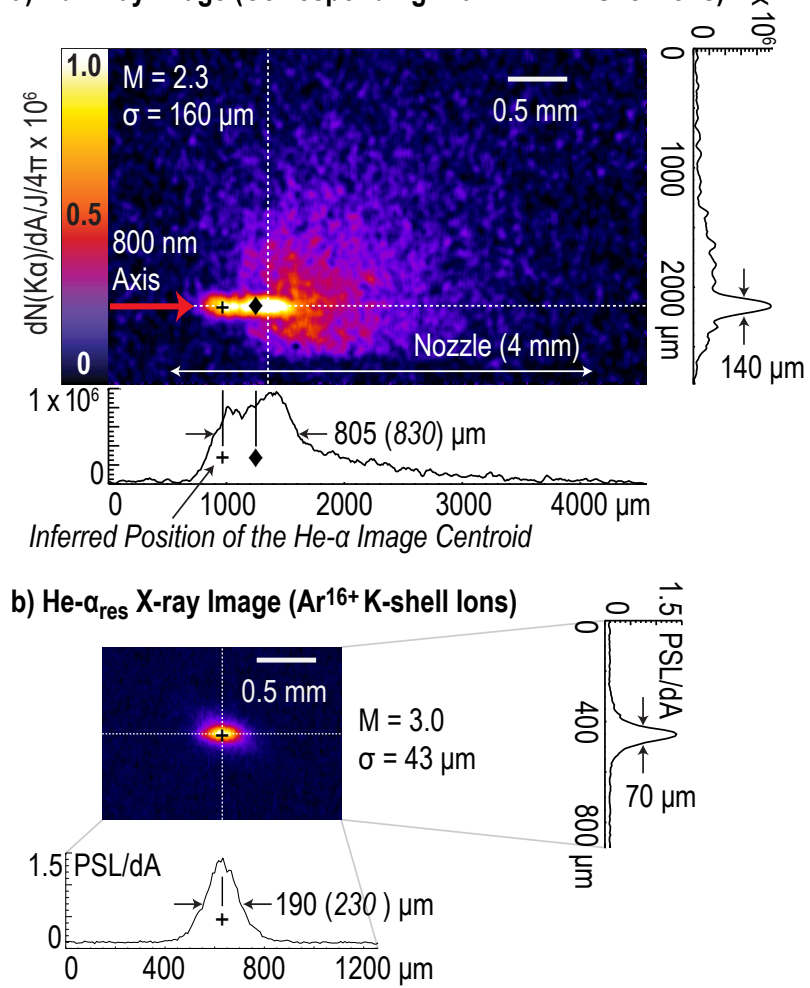

FIG. 2: (Color online) Time-integrated data from the monochromatic XRI diagnostics, showing the K-shell x-rays characteristic of two different Ar ionization states. Horizontal lengths in italics have been corrected for the parallax foreshortening caused by the observation angles as shown in Fig. 1. The position + of the He- $\alpha$ image centroid has been placed onto the $\mathrm{K} \alpha$ image using the offset shown in Fig. 3 . Locations of line profile sources are marked with dotted lines.

ture of $107 \mathrm{~mm}$ and used in the von Hamos geometry [16, 17] as shown in Fig. $1 \mathrm{~b}$ ). Centered at $3.05 \mathrm{keV}$, i.e. in the middle of the Ar K-shell spectrum between $\mathrm{K} \alpha$ and $\mathrm{He}-\alpha$, the Bragg angle was $\theta_{B}=36.3^{\circ}$ and the source to crystal distance was $\ell=171 \mathrm{~mm}$. The HOPG crystal had interplane spacing $2 d=0.67 \mathrm{~nm}$, a mosaic spread of $\gamma=0.8^{\circ}$ (ZYB grade), a width $w=25.4 \mathrm{~mm}$ and an integrated reflectivity $R_{\mathrm{int}}=3.0$ mrad. The x-rays were detected on absolutely calibrated Fujifilm BAS-SR imaging plate [18, 19]. Filtering consisted of $25 \mu \mathrm{m}$ of black Kapton (polyimide) to block visible light and $84 \mu \mathrm{m}$ of mylar to attenuate the $\mathrm{x}$-rays, yielding a transmission of $\tau_{F}(h v=3.05 \mathrm{keV})=0.13$. Given broadening effects inherent to the crystal $[16,17]$ and the $25 \mu \mathrm{m}^{2}$ pixel size of the image plate, the source monitor spectrometer had a minimum instrument function width of $1.3 \mathrm{eV}$ at $3.05 \mathrm{keV}$ for a resolution $\Delta E / E=4.3 \times 10^{-4}$.

To obtain polychromatic $\mathrm{x}$-ray imaging along the laser axis, the HOPG crystal was mounted above the horizontal lasertarget plane with the curved edge approximately parallel to the laser axis, as shown in Fig. 1 b). In this sagittal direction the von Hamos spectrometer instrument function is dominated by mosaic blurring, with a width given by $2 \gamma \ell \sin \theta_{B}=$ $2.8 \mathrm{~mm}$ [16]. Due to space limitations within the target chamber the image plate was mounted at an angle with respect to the horizontal best line-focus von Hamos imaging plane, further broadening the sagittal instrument function. In the dispersive direction, the mosaic blurring is much less [16], but

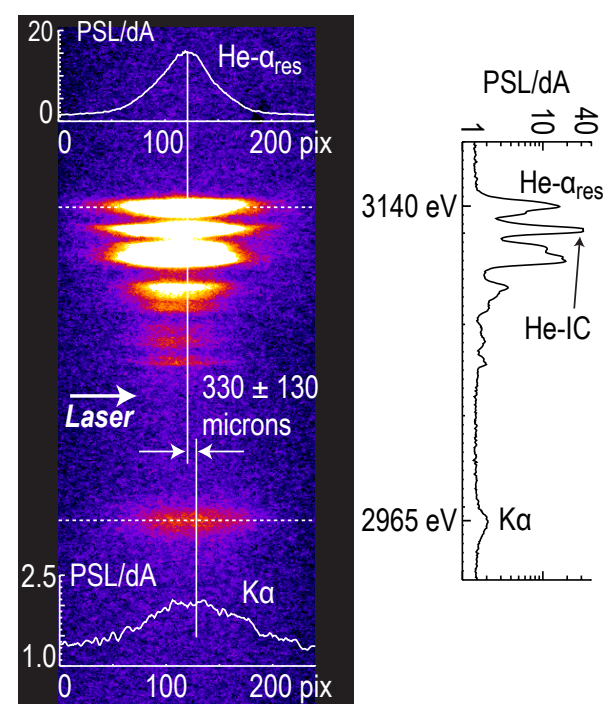

FIG. 3: (Color online) Time-integrated Ar K-shell x-ray spectrum. The von Hamos spectrometer geometry images in the non-dispersive (horizontal) direction with unit magnification. Locations of line profile sources are marked with dotted lines.

zeroth order imaging is convolved with the spectrum, complicating analysis. Given these considerations we restrict our imaging usage of the spectrometer data to comparing centroid positions from one portion of the spectrum to another in the sagittal direction, which tells us the relative location of different line-emission regions of plasma along the laser axis.

The monochromatic x-ray imagers consisted of two spherically bent quartz crystals, one each for $\mathrm{K} \alpha$ and $\mathrm{He}-\alpha_{\text {res }}$, with Fujifilm BAS-TR imaging plate detectors. Single spherically bent crystals can offer high spectral and spatial resolution when imaging $\mathrm{x}$-ray line emission; however, in order to maintain spatial resolution (i.e. to control off-axis geometric aberrations and distortions [20]) one must work at near normal incidence [21]. Every x-ray line of interest must therefore be paired with a crystal that can operate with Bragg angles $\theta_{B} \approx 90^{\circ}$ [22].

To image Ar K $\alpha$ at $2965 \mathrm{eV}$, we used a spherically bent quartz 200 crystal $(2 d=0.4246 \mathrm{~nm}$ [23]) with radius of curvature $r_{c}=40 \mathrm{~cm}$ at $\theta_{B}=80.0^{\circ}$. Solid angle was limited by a $1 \mathrm{~cm}$ diameter aperture cut into $0.5 \mathrm{~mm}$ thick $\mathrm{Al}$ substrate (opaque to $3 \mathrm{keV}$ ) that was placed $1 \mathrm{~mm}$ in front of the crystal. At the operating magnification of $M=2.3$ the analytically estimated resolution is $\sigma=160 \mu \mathrm{m}$ [22]. Likewise, for imaging $\mathrm{He}-\alpha_{\text {res }}$ at $3140 \mathrm{eV}$ we used a spherically bent quartz 201 crystal ( $2 d=0.3960 \mathrm{~nm}$ [23]) with radius of curvature $r_{c}=50$ $\mathrm{cm}$ at $\theta_{B}=85.1^{\circ}$. With the same aperture at the crystal and $M=3.0$, the analytically estimated resolution is $\sigma=43 \mu \mathrm{m}$. The working distances from the crystal to the plasma were $d_{o}$ $=280 \mathrm{~mm}$ for the 200 crystal and $d_{o}=330 \mathrm{~mm}$ for the 201 crystal.

Because the target material is a gas and therefore cannot be used in front of the detector as a K-edge $\mathrm{x}$-ray filter, proper collimation is key to obtaining good quality, high contrast $\mathrm{Ar}$ $\mathrm{x}$-ray images. Our XRI collimators, as shown in Fig. 1 a), were Al tubes with $6 \mathrm{~mm}$ thick walls. The $\mathrm{Al}$ was then lined with $1.6 \mathrm{~mm}$ of polytetrafluoroethylene (PTFE, $\mathrm{C}_{2} \mathrm{~F}_{4}$ ). This heavy plastic liner is opaque (transmission less than $10^{-8}$ ) up to photon energies of roughly $5 \mathrm{keV}$, which is sufficient 
to block $\mathrm{Al} \mathrm{K} \alpha$ fluorescence and bremsstrahlung originating from the interaction of laser-accelerated fast electrons with the $\mathrm{Al}$ collimator exterior. A further reduction in background noise was achieved by blocking all direct lines of sight from the imaging plates to the plasma with 1 inch thick lead bricks.

\section{SPATIAL DISTRIBUTION OF AR IONS}

As seen in Fig. 2, both $\mathrm{K} \alpha$ and He- $\alpha_{\text {res }}$ emission comes from regions of plasma that are elongated along the laser axis. The images shown are from two individual laser shots under similar laser conditions. $\mathrm{K} \alpha$ emission appears mostly along the laser axis as a long and narrow region measuring $830 \mu \mathrm{m}$ by $140 \mu \mathrm{m}$. This is substantially longer than the Rayleigh range $x_{R}=\pi r_{0}^{2} / \lambda=320 \mu \mathrm{m}$. Given that the resolution of the system is estimated to be $\sigma=160 \mu \mathrm{m}$, the height of the $\mathrm{K} \alpha$ feature can be interpreted as an approximate upper limit on the vertical extent of $\mathrm{K} \alpha$ emission. Additionally, a diffuse region of $\mathrm{K} \alpha$ emission extends beyond the main feature. The He$\alpha_{\text {res }}$ emission is much more localized than the $\mathrm{K} \alpha$ emission, measuring only $230 \mu \mathrm{m}$ long by $70 \mu \mathrm{m}$ tall, with a resolution of $\sigma=43 \mu \mathrm{m}$.

Rocking curves of the quartz crystal response to $\mathrm{Fe} \mathrm{K} \alpha \mathrm{x}$ rays were made offline by NSTec, Inc. The full width at half maximum (FWHM) of the rocking curves is $\Delta \theta=0.3^{\circ}$ for the quartz 200 crystal and $\Delta \theta=0.1^{\circ}$ for the quartz 201 crystal. Using the working distances specified above and neglecting the influence of source bandwidth, the FWHM fields of view are $d_{o} \Delta \theta=1.5 \mathrm{~mm}$ (200 crystal) and $0.58 \mathrm{~mm}$ (201 crystal). These estimates are moderately larger than the size of the corresponding features in Fig. 2. Stated differently, moderate chromatic vignetting [21] might be influencing image brightness at the margins of the images. A rigorous evaluation of the bandwidth of spherical crystal x-ray imaging of mm-scale Ar laser plasmas is being published separately [24].

Cross-calibration with the von Hamos x-ray spectrum allows us to place absolute units of photon emittance on the $\mathrm{K} \alpha$ image in Fig. 2 a). The analysis is straightforward because $\mathrm{K} \alpha$ is optically thin [25] and all volumes of emitting plasma contribute to the observed image. However, in the case of $\mathrm{He}-\alpha_{\text {res }}$, which is optically thick because of resonant photoabsorbtion, the image only includes that surface emission which is visible from the quartz crystal. Resonant photoabsorption by ions from a lower state $\ell$ to an upper state $u$ is proportional to $N_{\zeta, \ell}$, the number of ions with charge $\zeta$, and $B_{\zeta, \ell \rightarrow \zeta, u}$, the Einstein stimulated emission probability coefficient [3]. Significant He- $\alpha$ reabsorption occurs because the lower state $\ell$ of He- $\alpha$ is the heavily populated ground state. For simplicity, we therefore leave the He- $\alpha_{\text {res }}$ image in units of image plate exposure, or photo-stimulated luminescence (PSL).

The time-integrated von Hamos X-ray spectrum is shown in Fig. 3. Strong He-like and Li-like features dominate the highenergy end of the spectrum, while near-cold $\mathrm{K} \alpha$ is visible at the low-energy end of the spectrum. In the non-dispersive direction, lineouts of the $\mathrm{K} \alpha$ and $\mathrm{He}-\alpha_{\text {res }}$ features clearly show an offset of $330 \mu \mathrm{m}$ in the location of the $\mathrm{x}$-ray emitting centroids along the laser axis. $\mathrm{A} \pm 130 \mu \mathrm{m}$ one standard deviation uncertainty represents the variation in this offset measurement resulting from varying short pulse laser conditions from shot to shot. This centroid offset was used to infer the position of the He- $\alpha_{\text {res }}$ feature on the $\mathrm{K} \alpha$ image as shown in Fig. 2 a), as marked with a + symbol. The He- $\alpha$ comes from the leading edge of the $\mathrm{K} \alpha$ emission region with respect to the direction of laser propagation.

The spectral code FLYCHK [11] was used to generate a synthetic X-ray spectrum and correlate the X-ray photon energies with temperatures and ionization states. We used the same simulation parameters as described in Ref. [14]. FLY$\mathrm{CHK}$ results indicate that $\mathrm{K} \alpha$ emission at $2965 \mathrm{eV}$, as imaged here, is characteristic of $\mathrm{Ar}^{\approx 6+} \mathrm{M}$-shell ions with electron temperature $T_{e} \approx 20 \mathrm{eV}$. Similarly, the He- $\alpha_{\text {res }} \mathrm{X}$-rays imaged at $h v=3140 \mathrm{eV}$ are characteristic of $\mathrm{Ar}^{16+} \mathrm{K}$-shell ions in a plasma with electron temperature $T_{e} \gtrsim 100 \mathrm{eV}$.

\section{SUMMARY AND ACKNOWLEDGEMENTS}

We have demonstrated a technique for studying the extent of plasma ionization within a laser-irradiated Ar gas jet, providing fundamental physical information about laser-target interactions. Future application of this technique could include use on the National Ignition facility to help with ICF studies that require knowledge of the spatial extent of plasma at specific conditions during capsule implosions [26].

We would like to thank Jeff Koch, Kramer Akli and Daniel Hey at LLNL, and Guy Bennett at Sandia National Laboratory, for helpful discussions. Elena Baranova and Nino Pereira of EcoPulse, Inc. provided the x-ray imaging crystals. We also thank the staff of the Jupiter Laser Facility for their support. This work was performed under the auspices of the U.S. Department of Energy by the Lawrence Livermore National Laboratory, through the Institute for Laser Science and Applications, under Contract No. DE-AC5207NA27344. This work was also supported by DOE Grant No. DE-PS02-07ER07-28 (Plasma Physics Junior Faculty Award Program), LDRD Grant No. 08-ERI-002 and the LLNL Lawrence Scholar Program.
[1] T. Brabec, Strong Field Laser Physics (Springer, 2008), 1st ed., ISBN 038740077X.

[2] G. W. F. Drake, Springer Handbook of Atomic, Molecular, and Optical Physics (Springer, 2005), 2nd ed., ISBN 038720802X.

[3] D. Salzmann, Atomic Physics in Hot Plasmas (Oxford University Press, USA, 1998), ISBN 0195109309.

[4] S. H. Glenzer and R. Redmer, Reviews of Modern Physics 81, 1625 (2009).

[5] L. House, Astrophysical Journal Supplement Series 18, 21 (1969).
[6] W. BAMBYNEK, B. CRASEMANN, R. W. FINK, H. U. FREUND, H. MARK, C. D. SWIFT, R. E. PRICE, and P. V. RAO, Reviews of Modern Physics 44, 716 (1972).

[7] P. Neumayer, H. Lee, D. Offerman, E. Shipton, A. Kemp, A. Kritcher, T. Döppner, C. Back, and S. Glenzer, High Energy Density Physics 5, 244 (2009).

[8] M. Tanabe, T. Fujiwara, S. Fujioka, H. Nishimura, H. Shiraga, H. Azechi, and K. Mima, Review of Scientific Instruments 79 , 10E908 (2008).

[9] J. Koch, N. Izumi, L. Welser, R. Mancini, S. Haan, R. Lee, 
P. Amendt, T. B. Jr., S. Dalhed, K. Fujita, I. Golovkin, L. Klein, O. Landen, F. Marshall, D. Meyerhofer, H. Nishimura, Y. Ochi, S. Regan, T. Sangster, V. Smalyuk, et al., High Energy Density Physics 4, 1 (2008).

[10] S. B. Hansen, A. Y. Faenov, T. A. Pikuz, K. B. Fournier, R. Shepherd, H. Chen, K. Widmann, S. C. Wilks, Y. Ping, H. K. Chung, A. Niles, J. R. Hunter, G. Dyer, and T. Ditmire, Physical Review E 72, 036408 (2005).

[11] H. Chung, M. Chen, W. Morgan, Y. Ralchenko, and R. Lee, High Energy Density Physics 1, 3 (2005).

[12] H. Park, D. M. Chambers, H. Chung, R. J. Clarke, R. Eagleton, E. Giraldez, T. Goldsack, R. Heathcote, N. Izumi, M. H. Key, J. A. King, J. A. Koch, O. L. Landen, A. Nikroo, P. K. Patel, D. F. Price, B. A. Remington, H. F. Robey, R. A. Snavely, D. A. Steinman, et al., Physics of Plasmas 13, 056309 (2006).

[13] D. Porquet and J. Dubau, Astronomy and Astrophysics Supplement Series 143, 20 pages (2000).

[14] N. L. Kugland, P. Neumayer, T. Doppner, H. Chung, C. G. Constantin, F. Girard, S. H. Glenzer, A. Kemp, and C. Niemann, in PROCEEDINGS OF THE 17TH TOPICAL CONFERENCE ON HIGH-TEMPERATURE PLASMA DIAGNOSTICS (HTPD0) (AIP, Albuquerque, New Mexico (USA), 2008), vol. 79, pp. 10E917-3.

[15] A. Pak, G. Gregori, J. Knight, K. Campbell, D. Price, B. Hammel, O. L. Landen, and S. H. Glenzer, Review of Scientific Instruments 75, 3747 (2004).

[16] M. Urry, G. Gregori, O. Landen, A. Pak, and S. Glenzer, Journal of Quantitative Spectroscopy and Radiative Transfer 99, 636 (2006).

[17] A. P. Shevelko, I. I. Sobelman, and V. A. Slemzin, in Current Russian Research in Optics and Photonics: New Methods and Instruments for Space- and Earth-based Spectroscopy in XUV, UV, IR, and Millimeter Waves (SPIE, Russia, 1998), vol. 3406, pp. 91-108.

[18] A. L. Meadowcroft, C. D. Bentley, and E. N. Stott, Review of Scientific Instruments 79, 113102 (2008).

[19] I. J. Paterson, R. J. Clarke, N. C. Woolsey, and G. Gregori, Measurement Science and Technology 19, 095301 (2008).

[20] M. Born and E. Wolf, Principles of Optics: Electromagnetic Theory of Propagation, Interference and Diffraction of Light (Cambridge University Press, 1997), 6th ed., ISBN 0521639212.

[21] J. A. Koch, O. L. Landen, T. W. Barbee, P. Celliers, L. B. D. Silva, S. G. Glendinning, B. A. Hammel, D. H. Kalantar, C. Brown, J. Seely, G. R. Bennett, and W. Hsing, Appl. Opt. 37, 1784 (1998).

[22] J. A. Koch, Tech. Rep. UCRL-ID-129951, Lawrence Livermore National Laboratory (1998).

[23] G. M. Crankovic, ASM Handbook: Volume 10: Materials Characterization (Asm Handbook) (ASM International, 1986), 9th ed., ISBN 0871700166.

[24] N. L. Kugland, T. Döppner, A. Kemp, C. G. Constantin, S. H. Glenzer, and C. Niemann, (in publication) (2010).

[25] D. Duston, R. W. Clark, J. Davis, and J. P. Apruzese, Physical Review A 27, 1441 (1983).

[26] S. H. Glenzer, B. J. MacGowan, P. Michel, N. B. Meezan, L. J. Suter, S. N. Dixit, J. L. Kline, G. A. Kyrala, D. K. Bradley, D. A. Callahan, E. L. Dewald, L. Divol, E. Dzenitis, M. J. Edwards, A. V. Hamza, C. A. Haynam, D. E. Hinkel, D. H. Kalantar, J. D. Kilkenny, O. L. Landen, et al., Science p. science.1185634 (2010). 